\title{
In vitro Antimicrobial Activities of Vigna subterranea
}

\section{Ajiboye AA and Oyejobi GK $^{*}$}

Federal University, Department of Plant Sciences and Parasitology, Ekiti State, Nigeria

"Corresponding author: Oyejobi K, Greater Department of Biological Sciences, Osun State University, Osogbo, Osun State, Nigeria, Tel: 08132927279; E-mail: greateroyejobi@gmail.com

Received date: October 27, 2015; Accepted date: January 27, 2017; Published date: February 03, 2017

Copyright: (C) 2017 Ajiboye AA, et al. This is an open-access article distributed under the terms of the Creative Commons Attribution License, which permits unrestricted use, distribution, and reproduction in any medium, provided the original author and source are credited.

\begin{abstract}
The antibacterial assay of the seed of Vigna subterranea (Bambara Groundnut) on certain clinical isolates: Proteus mirabilis, Escherichia coli, Klebsiella aerogenes, Klebsiella ozaenae, Staphylococcus aureus and Pseudomonas aeruginosa was determined using the agar diffusion technique to investigate its potential use as antibacterial agent. The antimicrobial activity of the test organisms to the seed extract was screened by using the agar-well diffusion method. An inoculum suspension was swabbed uniformly to solidified $20 \mathrm{ml}$ Mueller-Hinton Agar (MHA) and the inoculum was allowed to dry for 5 minutes. Holes of $5 \mathrm{~mm}$ in diameter were made in the seeded agar using sterile cork-borer. Aliquot of $50 \mu \mathrm{l}$ from each seed extract was added into each well on the seeded medium and allowed to stand on the bench for 1 hour for proper diffusion and thereafter incubated at $37^{\circ} \mathrm{C}$ for 24 hour. The resulting inhibition zones were measured in millimeters $(\mathrm{mm})$. Negative controls using $50 \mu$ PBS were also run in the same manner and parallel to the treatments. These studies were performed in duplicate. The seed extracts of $V$. subterranea showed varying antimicrobial activities against the test organisms. Antimicrobial screening indicated that $V$. subterranea showed the highest $(39 \pm 2.55 \mathrm{~mm})$ inhibition against $E$. coli and $K$. ozanae at dilutions 1 and 2 respectively. The least activity was observed at dilutions 2 while $P$. mirabilis and $\mathrm{E}$. coli both had zones of inhibition of $8 \pm 0.00 \mathrm{~mm}$ and $8 \pm 1.41 \mathrm{~mm}$ respectively. Susceptibility testing using standard antibiotics (positive control) was also carried out. $P$. mirabilis, $E$. coli and $K$. aerogenes were susceptible to the entire tested antibiotics except Augumentin (AUG) and Amoxicillin (AMX). The diameters of the clear zones of inhibitions were measured and the result recorded to the nearest millimeters $(\mathrm{mm})$. The diameter of the zone was used as a measure of the susceptibility of the isolate and the diffusion rate of the seed extracts/antibiotics through the agar medium. The result of the study suggests that seed extract of $V$. subterranea can be used for the treatment of infections caused by the test organisms.
\end{abstract}

Keywords: In vitro; Vigna subterranean; Antibacterial assay; Clinical isolates; Bambara groundnut; Antibiotics; Seed extracts; Susceptibility

\section{Introduction}

According to the World Health Organization (WHO), a medicinal plant is any plant which in one or more of its organ contains substances that can be used for the synthesis of useful drugs [1]. Medicinal plants contain biologically active chemical substances such as saponins, tannins, essential oils, flavonoids, alkaloids and other chemical compounds [2] which have curative properties. These complex chemical substances of different compositions are found as secondary plant metabolites in one or more of these plants [3].

The rising prevalence of antibiotics resistant pathogenic microorganisms in the last decades raises the demand for finding new alternative antimicrobial agents. Therefore, the current study was carried out to evaluate the antimicrobial activity of the seed of Bambara plants which have potential of treating infectious diseases and with lesser side effects compared to the synthetic drug agents.

The aim and objectives of this research are to determine the antimicrobial activities of the seed extract of Vigna subterranea and to compare the antibacterial activities of the leaf extract of V. subterranea with antibiotics commonly used to treat infections of the test organisms.

\section{Literature Review}

Plants have been an important source of medicine since ancient times. Early written reports on the use of plants as medicine appeared about 2600 B.C. when plants were used as medicine by Sumerians and Akkaidians (Shoeb, 2006). Since then, plants have been used to treat ailments such as headaches, toothaches, stomach aches, diarrhea, wounds, tumors and sexually transmitted diseases (Van Wyk and Gericke, 2010; Wuyang, 2008; Khaleeliah, 2001; Von Koenen, 2001), just to mention a few. However, the potential of several plants as medicinal agents has not been fully characterized and established. This is due to the fact that most scientific studies carried out on plants focused on specific diseases, thereby revealing only a narrow spectrum of active compounds. This is often attributed to the limited resources, labor and time allocated for the study.

According to the World Health Organization (WHO), a medicinal plant is any plant which in one or more of its organ contains substances that can be used for the synthesis of useful drugs [1]. Medicinal plants contain biologically active chemical substances such as saponins, tannins, essential oils, flavonoids, alkaloids and other chemical compounds [2] which have curative properties against pathogenic bacteria and fungi species in humans.

The rising prevalence of antibiotics resistant pathogenic microorganisms in the last decades raises the demand for finding new alternative antimicrobial agents. Therefore, the current study was carried out to evaluate the antimicrobial activity of the seed of 
Page 2 of 4

Bambara plants which have potential of treating infectious diseases and with lesser side effects compared to the synthetic drug agents.

\section{Methodology Applied}

The research was carried out between July and August, 2014 at the Microbiology Laboratory of the Department of Biological Sciences, Osun State University Osogbo, Osun state (Latitude $7.5^{\circ} \mathrm{N}$ and Longitude $4.5^{\circ} \mathrm{E}$, at $246 \mathrm{~m}$ elevation). The seeds of Bambara groundnut (Vigna subterranea) with accession number TVsu 283 (Figure 1) were collected from International Institute of Tropical Agriculture (IITA), Ibadan, Oyo State. The seeds were treated by soaking in $0.02 \%$ sodium hypochlorite for 2 minutes and washed five times with sterilized distilled water [4].

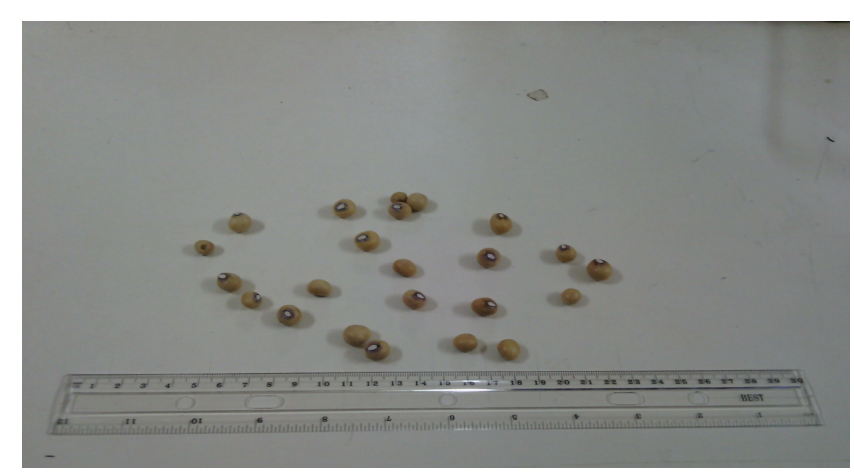

Figure 1: Seeds of $V$. subterranea (TVsu 283).

The seeds were air-dried at room temperature over a period of three days. The dried seeds were pulverized to dry powder using an electric blender. The powder was soaked in ethanol (seed material to solvent ratio was $1: 10, w / v)$ for 72 hours using cold extraction methods with regular agitation [5]. The extract was evaporated to dryness using steam bath and stored in sterile universal bottle as described by Sliver et al. The extract was filtered using a muslin cloth and the filterate was evaporated to dryness using steam water bath at $78^{\circ} \mathrm{C}$. The extract was then re-constituted in Dimethy Sulphoxide (DMSO).

The filtrate was sterilized by filtering through Millipore membrane filter of $0.45 \mu \mathrm{m}$ pore size. The extract was then tested for sterility by introducing $2 \mathrm{ml}$ of this supposed sterile extract into $10 \mathrm{ml}$ of sterile nutrient broth. Incubation was done at $37^{\circ} \mathrm{C}$ for 24 hours. A sterile extract was indicated by absence of turbidity or clearness of the broth after the incubation period. It was stored in the refrigerator prior to use.

The test organisms were 6 clinical isolates of Proteus mirabilis, Escherichia coli, Klebsiella aerogenes, Klebsiella ozaenae, Staphylococcus aureus, and Pseudomonas aeruginosa obtained through due process from the stock culture of Microbiology Laboratory of the Department of Medical Microbiology, Ladoke Akintola University of Technology, Osogbo.

The bacterial and the fungal isolates were grown in nutrient broth for 18 hour at $35^{\circ} \mathrm{C}$. The inoculum load was adjusted to $1 \times 10^{-3} \mathrm{cfu} / \mathrm{ml}$ via serial dilution method prior to use [5]. The antimicrobial activity of the test organisms to the seed extract was screened by using the agarwell diffusion method (Perez et al.). An inoculum suspension was swabbed uniformly to solidified $20 \mathrm{ml}$ Mueller-Hinton Agar (MHA) and the inoculum was allowed to dry for $5 \mathrm{~min}$. Holes of $5 \mathrm{~mm}$ in diameter were made in the seeded agar using sterile cork-borer [6]. Aliquot of $50 \mu \mathrm{l}$ from each seed extract was added into each well on the seeded medium and allowed to stand on the bench for 1 hour for proper diffusion and thereafter incubated at $37^{\circ} \mathrm{C}$ for 24 hour [6]. The resulting inhibition zones were measured in millimeters ( $\mathrm{mm}$ ). Negative controls using $50 \mu \mathrm{l}$ PBS were also run in the same manner and parallel to the treatments. These studies were performed in duplicate [6].

The diameters of the clear zones of inhibitions were measured and the result recorded to the nearest millimeters $(\mathrm{mm})$. The diameter of the zone is related to the susceptibility of the isolate and to the diffusion rate of the seed extracts through the agar medium [5]. Statistical analysis of all tests was carried out using SPSS 15.0 design. Data was analyzed with Analysis of Variance (ANOVA) at $\mathrm{P}<0.05$ level. Tests were conducted in duplicates and values given as mean \pm standard error.

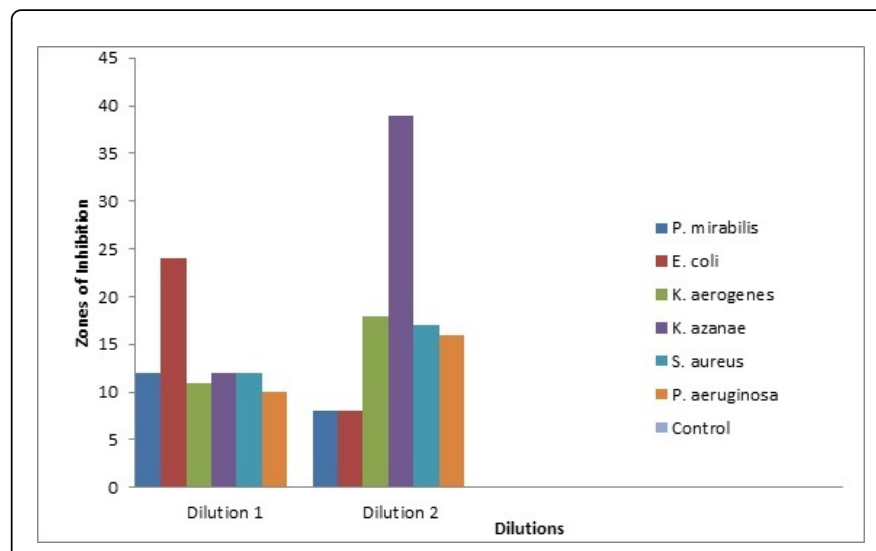

Figure 2: Mean diameter of zones of inhibition ( $\mathrm{mm})$. Values are mean $\pm \mathrm{SD}$ of two replicates. Diameter of cork-borer $=5 \mathrm{~mm}$.

\section{Results}

\section{Antimicrobial activities of the seeds of Bambara}

The seed extracts of $V$. subterranea showed varying antimicrobial activities against the test organisms: Proteus mirabilis, Escherichia coli, Klebsiella aerogenes, Klebsiella ozanae, Staphylococcus aureus and Pseudomonas aeruginosa. Figure 2 shows that all the test organisms were susceptible to the Bambara seed extract although with varying activities. K. ozanae had the highest activity of $39 \pm 2.55 \mathrm{~mm}$ at $10^{-2}$ dilution, followed by $E$. coli with activity of $24 \pm 2.83 \mathrm{~mm}$ at $10^{-1}$ dilution, then $K$. aerogenes, $S$. aureus and $P$. aeruginosa with activities of $18 \pm 3.00 \mathrm{~mm}, 17 \pm 1.83 \mathrm{~mm}$ and $16 \pm 1.37 \mathrm{~mm}$ respectively at $10^{-2}$ dilution. Following these are $P$. mirabilis, $K$. ozanae and $S$. aureus having $12 \pm 0.50 \mathrm{~mm}, 12 \pm 2.12 \mathrm{~mm}$ and $12 \pm 1.57 \mathrm{~mm}$ respectively at $10^{-1}$ dilution. Following closely are those shown by $K$. aerogenes and $P$. aeruginosa with activities of $11 \pm 1.00 \mathrm{~mm}$ and $10 \pm 1.87 \mathrm{~mm}$ respectively at 10-1 dilution. The lowest activity was observed in $P$. mirabilis and $E$. coli with activities of $8 \pm 0.00 \mathrm{~mm}$ and $8 \pm 1.41 \mathrm{~mm}$ respectively at 10-2 dilution. 
Page 3 of 4

\section{Susceptibility testing using standard antibiotics}

Table 1 shows the susceptibility testing using standard antibiotics (positive control). P. mirabclis, $E$. coli and $K$. aerogenes were susceptible to the entire tested antibiotics except Augumentin (AUG) and Amoxicillin (AMX). S. aureus was only susceptible to Gentamycin (GEN) with zone of inhibition of $7 \pm 0.29 \mathrm{~mm}$; P. aeruginosa was only susceptible to Ofloxacin (OFL) and Nitrofurantoin (NIT) with zones of inhibition of $9 \pm 0.22 \mathrm{~mm}$ and $4 \pm 0.50 \mathrm{~mm}$ respectively; while $K$. ozanae was susceptible only to Tetracycline (TET), Ofloxacin (OFL) and Nalidixic acid (NAL) with zones of inhibition of $2 \pm 1.00 \mathrm{~mm}, 13 \pm$ $0.15 \mathrm{~mm}$ and $5 \pm 0.40 \mathrm{~mm}$ respectively. All the test organisms were resistant to Augumentin (AUG) and Amoxicillin (AMX).

\begin{tabular}{|c|c|c|c|c|c|c|c|}
\hline Antibiotic Discs & P. mirabilis & E. coli & K. aerogenes & K. ozanae & S. aureus & $P$. aeruginosa & Control \\
\hline TET & $4 \pm 0.50$ & $7 \pm 0.29$ & $7 \pm 0.29$ & $2 \pm 1.00$ & 0 & 0 & 0 \\
\hline AUG & 0 & 0 & 0 & 0 & 0 & 0 & 0 \\
\hline OFL & $11 \pm 0.18$ & $4 \pm 0.50$ & $5 \pm 0.40$ & $13 \pm 0.15$ & 0 & $9 \pm 0.22$ & 0 \\
\hline NAL & $8 \pm 0.25$ & $7 \pm 0.29$ & $6 \pm 0.33$ & $5 \pm 0.40$ & 0 & 0 & 0 \\
\hline GEN & $7 \pm 0.29$ & $8 \pm 0.25$ & $8 \pm 0.25$ & 0 & $7 \pm 0.29$ & 0 & 0 \\
\hline NIT & $2 \pm 1.00$ & $7 \pm 0.29$ & $7 \pm 0.29$ & 0 & 0 & $4 \pm 0.50$ & 0 \\
\hline СОT & $9 \pm 0.22$ & $8 \pm 0.25$ & $8 \pm 0.25$ & 0 & 0 & 0 & 0 \\
\hline AMX & 0 & 0 & 0 & 0 & 0 & 0 & 0 \\
\hline
\end{tabular}

Values are mean \pm SD of two replicates; TET: Tetracycline $30 \mu \mathrm{g}$; AUG: Augumentin $30 \mu \mathrm{g}$; NIT: Nitrofurantoin $300 \mu \mathrm{g}$; NAL: Nalidixic acid $30 \mu \mathrm{g}$; GEN: Gentamycin 10

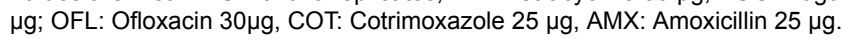

Table 1: Susceptibility testing using standard antibiotics-positive control (mm).

\section{Discussion}

E. coli showed the highest activity on the extract at $10^{-1}$ dilution with zone of inhibition of $24 \pm 2.83 \mathrm{~mm}$, followed by $K$. ozanae, $S$. aureus and P. mirabilis having zones of inhibition of $12 \pm 2.12 \mathrm{~mm}, 12$ $\pm 1.57 \mathrm{~mm}$ and $12 \pm 0.50 \mathrm{~mm}$ respectively. $K$. aerogenes had zone of inhibition of $11 \pm 1.00 \mathrm{~mm}$, while the lowest activity at 10-1 dilution was found in P. aeruginosa with activity of $10 \pm 1.87 \mathrm{~mm}$.

$K$. ozanae showed the highest activity on the extract at $10^{-2}$ dilution. The zone of inhibition was $39 \pm 2.55 \mathrm{~mm}$, followed by $K$. aerogenes with $18 \pm 3.00 \mathrm{~mm}, S$. aureus with $17 \pm 1.83 \mathrm{~mm}, P$. aeruginosa with 16 $\pm 1.37 \mathrm{~mm}$, and the lowest activity was found in P. mirabilis and E. coli both with zones of inhibition of $8 \pm 0.00 \mathrm{~mm}$ and $8 \pm 1.41 \mathrm{~mm}$ respectively.

With the exception of $E$. coli and P. mirabilis which were more susceptible to the Bambara seed extract at a lower dilution (10-1 dilution) with activities of $24 \pm 2.83 \mathrm{~mm}$ and $12 \pm 0.50 \mathrm{~mm}$ respectively, the results indicated that the Bambara seed extract is most effective at higher dilutions, i.e. the higher the dilution, the more effective the seed extract becomes. This was observed as all of the test organisms had an increase in their activities as the dilution increased from 10-1 to 10-2, except $E$. coli and $P$. mirabilis. $K$. aerogenes, $K$. ozanae, $S$. aureus and $P$. aeruginosa had their activities increased from $11 \pm 1.00 \mathrm{~mm}, 12 \pm 2.12 \mathrm{~mm}, 12 \pm 1.57 \mathrm{~mm}$ and $10 \pm 1.87 \mathrm{~mm}$ respectively to $18 \pm 3.00 \mathrm{~mm}, 39 \pm 2.55 \mathrm{~mm}, 17 \pm 1.83 \mathrm{~mm}$ and $16 \pm$ $1.37 \mathrm{~mm}$ respectively.

Several previous findings [7-11] reported that Gram-negative bacteria were not susceptible to plant extracts when compared to Gram-positive bacteria. The resistance of Gram-negative bacteria towards antibacterial substances is related to lipopolysaccharides in their outer membrane [12]. This is in contrast with the obtained results since most of the extracts showed prominent activity against gramnegative bacteria.

Most of the test organisms had higher zones of inhibition when compared to the commonly used antibiotic. From the results of the susceptibility testing using standard antibiotics, it was observed that all the test organisms are resistant to both AUG and AMX with none having zones of inhibition. It was also observed that $S$. aureus is resistant to all the commonly used antibiotics except GEN with activity of $7 \pm 0.29 \mathrm{~mm}$, while $P$. aeruginosa is only susceptible to OFL and NIT with activities of $9 \pm 0.22 \mathrm{~mm}$ and $4 \pm 0.50 \mathrm{~mm}$ respectively.

\section{Conclusions}

The study of the antimicrobial activities of Bambara seeds showed that Bambara seed extracts can be used for the treatment of various gastroenterological diseases, wound infections, septicemia, pneumonias and many other infections implicated by the test organisms. Bambara seeds could be useful as cheap source of herbal drugs and food supplements. In conclusion, the results obtained confirm the folkloric anticipation of the antimicrobial effectiveness and the therapeutic applications of Bambara plant. It is thus recommended that the medicinal importance of Bambara seeds be exploited by utilizing them in preparing drugs, teas, antiseptic creams, and antiseptic soaps and so on.

\section{References}

1. World Health Organization (WHO) (1977) Resolution-promotion and development of training and research in traditional medicine. 30: 49.

2. Sofowora A (1996) Medicinal Plant and Traditional Medicine in Africa. (2nd edn) Spectrum Books, Ibadan, Nigeria: pp 112.

3. Kayode AA, Kayode OT (2011) Some medicinal values of telfairia occidentalis: A review. Am J Biochem Mol Biol 1: 30-38. 
Citation: Ajiboye AA, Oyejobi GK (2017) In vitro Antimicrobial Activities of Vigna subterranea. J Antimicrob Agents 3: 132. doi: $10.4172 / 2472-1212.1000132$

Page 4 of 4

4. Gholami A, Shahsavani S, Nezarat S (2009) The effect of plant growth promoting rhizobacteria on germination, seedling Growth and yield of maize. World Acad Sci Eng Technol 49: 19-24.

5. Gbadamosi IT, Alia AE, Okolosi O (2012) In-vitro antimicrobial activities and nutritional assessment of roots of ten Nigerian vegetables. NY Sci J 5 : 234-240.

6. Maher O, Mohamad S, Mohammad A, Enas A, Hanee A, et al. (2012) Antimicrobial activity of crude extracts of some plant leaves. Res J Microbiol 7: 59-67.

7. Branter A, Male Z, Pepelinfak S, Atolic A (1996) Antimicrobial activity of Paliurus spina. J Ethnopharmacol 52: 119-122.

8. Nostro A, Germano MP, Angelo VD, Marino A, Cannatelli MA (2000) Extraction methods and bioautography for evaluation of medicinal plant antimicrobial activity. Lett Applied Microbiol 30: 379-384.
9. Ojala T, Remes S, Haansuu P, Vuorela H, Hiltunen R, et al. (2000) Antimicrobial activity of some coumarin containing herbal plants growing in Finland. J Ethnopharmaco 73: 299-305.

10. Sawer IK, Berry MI, Ford JL (1997) Effect of medium composition, agitation and presence of EDTA on the antimicrobial activity of cryptolepine. Lett. Applied Microbiol 25: 207-211.

11. Silver OA, Cabrita T, Pimentel M, Diniz A, Gomes E (1997) Antimicrobial activity of Guinea Bissau traditional remedies. J Ethnopharmacol 50: 55-59.

12. Gao Y, van Belkum MJ, Stiles ME (1999) The outer membrane of Gramnegative bacteria inhibits antibacterial activity of Brochocin-C. Applied Environ Microbiol 65: 4329-4333. 